\title{
VAC.2I - Evaluation of humoral immune response against Streptococcus pneumoniae elicited by vaccination of mice with a recombinant influenza virus
}

Kimberly Freitas Cardoso ${ }^{1 *}$; Beatriz Senra Álvares da Silva Santos²; Eliane Namie Miyaji; Marcelo Antônio Pascoal Xavier ${ }^{1}$; Pedro Augusto Alves ${ }^{1}$; Marco Antônio da Silva Campos ${ }^{1}$; Alexandre de Magalhães Vieira Machado ${ }^{1}$.

1Fiocruz/CPqRR;

2UFMG - Universidade Federal de Minas Gerais;

3Instituto Butantan.

Introduction: Streptococcus pneumoniae is a major cause of pneumonia and meningitis, resulting in great morbidity and mortality worldwide. The licensed pneumococcal conjugate vaccines, despite reducing the death rates of pneumococcal infections, are expensive and confer protection only against the serotypes included in vaccine formulation. Therefore, the emergence of new circulating serotypes is a non-negligible risk, arguing favorably for the development of new vaccines capable to elicit broad range protection. Thus, in the present work we generated a recombinant influenza virus carrying a pneumococcal surface protein (named X for patent issues), aiming the development of a vaccine able to induce broad range immune response against $S$. pneumoniae.

Objective: This work aims to evaluate the ability of a vaccination protocol using a recombinant influenza virus encoding a $S$. pneumoniae protein to induce specific anti-pneumococcus humoral immune response in murine model.

Methodology: The recombinant influenza virus was constructed by reverse genetics and characterized by PCR, sequencing and titration on MDCK cells. Female C57BL/6 mice ( $n=6 /$ group, license number LW-9/17) were inoculated twice with recombinant virus and/or recombinant protein or sterile PBS. At previously established time points, blood samples were collected and specific anti-X protein antibodies in serum of immunized mice were assessed by ELISA. The results were submitted to analysis of variance followed by the Tukey multiple comparisons test, with statistical significance $\rho<0.05$.

Results: To date, our results showed that our vaccination protocol has induced high levels of specific anti-X IgG seric antibodies. Moreover, both IgG1 and IgG2c isotype were detected in the sera of immunized mice, with significantly higher titers of IgG2c than IgG1. It is noteworthy that IgG2c is the subclasse of IgG which has the highest ability to mediate the protection against $S$. pneumoniae by leading the complement deposition on the surface of the bacteria, resulting in bacterial death by IgG-mediated opsonophagocytosis.

Conclusion: Overall, our results indicate that immunization with this vaccination protocol was able to induce specific humoral immune response in mice and has a great potential to be used in the development of new vaccines against S. pneumoniae.

Keywords: Streptococcus pneumoniae; Recombinant influenza virus; Humoral immune response 